\title{
Pre-Implantation Gender Selection: Family Balancing in Jordan
}

\author{
Amer Mahmoud Sindiani (ID) \\ Faheem Zayed (D) ${ }^{2}$ \\ Eman Hussein Alshdaifat ${ }^{3}$ \\ Hasan M Rawashdeh (D) \\ Wesam Al-Woshah ${ }^{4}$ \\ Nada Zayed ${ }^{5}$ \\ 'Department of Obstetrics and \\ Gynecology, Faculty of Medicine, Jordan \\ University of Science and Technology, \\ Irbid, Jordan; ${ }^{2}$ Department of Obstetrics \\ and Gynecology, Irbid Speciality Hospital, \\ Irbid, Jordan; ${ }^{3}$ Department of Obstetrics \\ and Gynecology, Yarmouk University, \\ Irbid, Jordan; ${ }^{4}$ Department of Obstetrics \\ and Gynecology, IBN Al-Nafis Hospital, \\ Irbid, Jordan; ${ }^{5}$ Department of \\ Dermatology, Albalqa Applied University, \\ Salt, Jordan
}

Correspondence: Amer Mahmoud Sindiani Department of Obstetrics and Gynecology, Faculty of Medicine, Jordan University of Science and Technology, PO Box: 3030, Irbid, 22II0, Jordan

Tel +962796025538

Email amsindiani0@just.edu.jo
Purpose: To find out whether couples with predominant female offspring have more chances to produce more female embryos during non-medical pre-implantation genetic diagnosis (PGD) for male sex selection.

Methods: A total of 125 couples who had three or more female offspring and underwent nonmedical PGD for male sex selection between 2015 and 2019 were included. Nuclear DNA was analyzed by fluorescent in situ hybridization (FISH). Two-chromosome (X, Y), 3-chromosome $(21, \mathrm{X}, \mathrm{Y})$, and 5-chromosome $(13,18,21, \mathrm{X}, \mathrm{Y})$ probes were used for FISH. The standard protocol was followed for sperm processing and embryo culture for IVF and PGD.

Results: In $83.2 \%$ of the couples, the ratio of female embryos was higher than male embryos. Independent sample $t$-test showed that there is no significant difference between equal and unequal embryonic groups in patients' age, husbands' age, sperm count, sperm motility, total male embryos, total female embryos, normal male embryos, and normal female embryos. For patients with positive pregnancy outcome, $84.6 \%$ had unequal embryonic ratio while $15.4 \%$ had equal embryonic ratio. Similarly, patients who were treated by short protocol had $85 \%$ of unequal embryonic ratio and $15 \%$ had equal ratio.

Conclusion: A greater variability in the female to male embryonic ratio is produced in couples having predominantly female offspring and seeking non-medical PGD for male sex selection.

Keywords: embryos, infertility, IVF, PGD

\section{Introduction}

The first human live births which used pre-implantation genetic diagnosis (PGD) during embryonic development to identify the presence of lethal genetic diseases in cycles of assisted reproduction were introduced in $1990 .{ }^{1}$ This treatment has gained momentum in assisted reproductive technology (ART). The DNA-based PGD treatment strategy has opened a new avenue in the treatment of infertility and embryonic sex-selection for medical and non-medical reasons. One of the most trusted DNA-based diagnostic procedures in ART is PGD. This technique helps physicians to select the unaffected embryos for uterine transfer in cases of patients who are carriers of single gene disorders or patients with structural chromosomal abnormalities. ${ }^{2}$ In addition to single gene defect detections, technical advances in single-cell genetic analysis, including single nucleotide polymorphism (SNP) array, comparative genomic hybridization (CGH), and whole genome amplification (WGA), may improve diagnostic precision and permit useful pre-implantation genetic screening (PGS) in patients with recurrent pregnancy loss and unexplained in vitro fertilization (IVF) treatment failure, where chromosomal errors are the 
result of potentially de novo mutations or meiotic and mitotic aberrations. ${ }^{3-7}$ Additionally, this technique is being used for non-medical reasons, enabling the selection of embryos of the desired sex. While there is appreciation for the use of PGD for medical reasons, the use of this technique for non-medical reasons has raised serious ethical concerns because non-medical sex selection can cause gender imbalance in communities and may cause destruction of unwanted normal embryos. ${ }^{8-10}$ Gender variety, or "family balancing" as it is sometimes known, is a form of PGD that is undertaken in families in which all offspring are of the same gender. Patients pursuing this option are interested in the unique experience of raising a child of the unrepresented gender. ${ }^{11,12}$ The ethics committees of both the American Congress of Obstetricians and Gynecologists (ACOG) and the American Society for Reproductive Medicine (ASRM) have established acceptable medical justifications for sex selection. However, the committees differ on the ethics of gender selection for non-medical indications. ${ }^{13}$

There are limited studies investigating the biological characteristics of couples having dominantly female or male offspring. In one retrospective study, 122 IVF/PGD cases from 2004 to 2009 were studied in which the PGD success rate for gender selection was found to be significantly lower compared to conventional IVF. ${ }^{14}$ In another PGD study, 276 patients of US nationality were reviewed retrospectively. This study found no biological significance in couples previously having children of a particular gender that would indicate a greater likelihood of producing embryos of that same gender. ${ }^{15}$ In a recent study, pre-implantation embryonic sex ratio was studied retrospectively in nine Israeli women who underwent PGD for non-medical sex selection. The results revealed a lower percentage of the desired embryos obtained in PGD compared to IVF. However, the result of this study cannot represent the rest of the population due to the very low sample size. Hence, in the present study, we aimed at finding the embryonic sex ratio in 125 couples who had three or more female offspring and underwent PGD for a non-medical reason.

\section{Materials and Methods}

In this retrospective study, 125 couples with three or more female offspring and those who underwent PGD for nonmedical sex selection (XY) between 2015 and 2019 were included. The patients' details were collected from the repositories at King Abdullah University Hospital/Jordan
University of Science and Technology and Ibn AlNafis Hospital.

All patients were counseled by their medical providers and then provided informed consent to participate in IVF/PGD. Patients underwent ovarian stimulation with gonadotropins using GnRH-agonist, few antagonist cases; luteal-phase down regulation short or long protocol to prevent premature luteinization of follicles. Serial monitoring was performed by IVF consultants for controlled ovarian stimulation through hormones and ultrasound analysis. When at least 2-3 follicles measured more than $18 \mathrm{~mm}$ in diameter, human chorionic gonadotropin (HCG) (5000-10,000 IU intramuscularly) was administered by intramuscular injection followed by transvaginal ultrasound-guided oocyte retrieval 36 hours later. In all cases, intra-cytoplasmic sperm injection (ICSI) was performed. All patients had embryo biopsy performed on day 3 after oocyte retrieval, by direct aspiration of a single blastomere through an opening created by laser degradation of the zona pellucida. The biopsied blastomere was fixed to a glass microscope slide and the cytoplasm was removed before PGD analysis, which includes nuclear DNA analysis by fluorescent in situ hybridization (FISH) using 2-chromosome (X, $\mathrm{Y})$, 3-chromosome (21, X, Y), and 5-chromosome (13, $18,21, \mathrm{X}, \mathrm{Y})$ probes.

Fluorescent probe binding to the centromeric region of the $\mathrm{X}$ chromosome and the $\mathrm{Y}$ chromosome was based on heat control denaturation and hybridization protocol targeted to nuclease at the $\mathrm{S}$ phase. Each nucleus is fixed to a glass microscope slide marked with a circle and embryo number using carbide glass pen. Targeted nucleic acid probes to the centromeric region of the chromosomes were attached to fluorochromes with different light emission frequencies, where each targeted probe for a chromosome resulted in a specific color referenced in the kit (Visis Abbott XY Probe Kit and PGT 5Probes Kit). ${ }^{16}$

Visual observations were obtained by well-trained technicians, as well as software capturing for result confirmation and archiving. The evaluation was subjected to visualization of the fluorochromes using filter light emitted fluorescent microscope based on the fluorescent range of the fluorochromes used in the binding probes.

Two different colors are observed for chromosomes $\mathrm{X}$ and $\mathrm{Y}$. If the same color of chromosome $\mathrm{X}$ is observed then the embryo is classified as $\mathrm{XX}$, referring to the female 
embryo. Vice versa, if a different color is observed, then the embryo would be classified as a male embryo.

Aneuploidy and euploidy is based on the number of colors observed. If two colors are observed, then the embryo is euploidy suitable for transfer, but if more than two colors are observed the embryo is classified as aneuploidy and thus not suitable for transfer.

A report was issued containing the case details, slide number, embryo number, result of the analysis, a photo of the visualized nucleus, and the color of the imitation probes. Aneuploidy and euploidy were written into the result row of the referred number of embryos. Artifacts and out of nucleus boundaries fluorescent was not considered in the result.

PGD results were evaluated by geneticists, embryologists and the IVF consultant responsible for embryo transfer on day 4 or day 5 of the embryo development. Patients were counseled about the FISH results prior to the transfer of the embryos.

The patients who could reach ovum pick-up stage and at least had complete molecular diagnosis of one embryo followed by day 3 blastomere biopsy were included in the study. The standard protocol was followed for sperm processing and embryo culture for IVF and PGD.

Statistical analysis was performed using IBM SPSS version 21. Descriptive statistics were calculated to describe the participant demographic characteristics. Ratio statistics were performed to find out whether greater variability in the sex ratio exists in the study population or not. Independent sample $t$-test and chi-square test were performed for continuous and categorical variables, respectively. The level of significance was set at $\mathrm{p}<0.05$. This study was approved by the Institutional Review Board of the Jordan University of Science and Technology (JUST)/King Abdullah University Hospital (KAUH) (36/121/2019). Patient consent was waived as this retrospective study involves electronic medical records review and analysis was performed on deidentified data. Patient data privacy and confidentiality were maintained as this study was conducted in compliance with the ethical standards of the Declaration of Helsinki.

\section{Results}

The results for the couples (with three or more female children), who underwent PGD for sex selection, were 289 male and 296 female embryos. The mean age of the patients and their husbands was 35 years and 41 years, respectively. The youngest patient was 25 years old, and the oldest patient was 47 years old. Similarly, the age of the youngest husband was 28 and the oldest husband was 57 years. Out of 125 patients, $83.2 \%$ were with unequal embryonic ratio and $16.8 \%$ had equal embryonic ratio. The median embryonic ratio was found to be 1 . Patients within $20 \%$ of median inclusion accounted for only $24.2 \%$ of those present (Table 1). This indicates larger variability in the ratio of female to male embryos in the study population.

The patients were divided into two groups based on the ratio of total male to total female embryos. Independent sample $t$-test showed that there is no significant difference between equal and unequal embryonic groups in patients' age, husbands' age, sperm count, sperm motility (\%), number of eggs, number of embryos, total male embryos, total female embryos, normal male embryos, and normal female embryos (Table 2). Chi-square test was performed to check the relationship between the type of protocol, embryonic ratio and pregnancy outcome (for total embryos). For patients with positive pregnancy outcome, $84.6 \%$ had an unequal embryonic ratio while $15.4 \%$ had an equal embryonic ratio. Similarly, patients who were treated by short protocol had $85.0 \%$ of unequal embryonic ratio and $15.0 \%$ had an equal ratio (Table 3). Similarly, independent sample $t$-test and chi-square test were performed for normal embryos to check whether any significant relationships exist between variables (Supplementary Tables 1 and 2 ).

\section{Discussion}

We found larger variability in the ratio of female to male embryos in couples who had three or more girl children and underwent non-medical PGD for male sex selection. The ratio statistics performed in this population confirmed the fact that couples previously having three or more girl children predominantly produce embryos of the same gender (female embryos) during PGD programs for male sex selection. This is consistent with the finding of a retrospective study performed on a large series of PGD procedures for gender selection in a wide geographical region in the USA. ${ }^{15}$

Table I The Participants' Characteristics

\begin{tabular}{|l|l|}
\hline Characteristics & Value \\
\hline Total female embryo & 296 \\
Total male embryo & 289 \\
Patients with equal embryonic ratio (\%) F:M & 16.8 \\
Patients with unequal embryonic ratio (\%) F:M & 83.2 \\
Median embryonic ratio F:M (N=95, Missing data=30): & 1.00 \\
Median (minimum, maximum) & $(0,8)$ \\
Co-efficient of concentration (within 20\% of median & $24.2 \%$ \\
inclusion) & \\
\hline
\end{tabular}


Table 2 Independent Sample $t$-Test (for Total Embryos)

\begin{tabular}{|l|l|l|l|}
\hline Variable & $\begin{array}{l}\text { Equal } \\
\text { Ratio } \\
\text { Mean } \pm \text { SD }\end{array}$ & $\begin{array}{l}\text { Unequal } \\
\text { Ratio } \\
\text { Mean } \pm \text { SD }\end{array}$ & p-value \\
\hline Patient age & $33.94 \pm 3.39$ & $35.19 \pm 4.48$ & 0.273 \\
Male partner age & $40.37 \pm 4.89$ & $41.45 \pm 5.66$ & 0.472 \\
Sperm count (million) & $89.35 \pm 45.5$ & $78.62 \pm 50.87$ & 0.417 \\
Motility (\%) & $58.64 \pm 9.65$ & $52.10 \pm 15.28$ & 0.091 \\
Eggs (number) & $11.70 \pm 4.76$ & $12.77 \pm 7.07$ & 0.550 \\
Embryos (number) & $6.35 \pm 2.34$ & $6.82 \pm 3.11$ & 0.552 \\
Total male embryos (number) & $2.64 \pm 1.16$ & $2.84 \pm 1.84$ & 0.672 \\
Total female embryos (number) & $2.64 \pm 1.16$ & $2.83 \pm 2.03$ & 0.715 \\
Normal male embryos (number) & $2.25 \pm 1.18$ & $2.60 \pm 1.55$ & 0.380 \\
Normal female embryos & $2.31 \pm 1.13$ & $2.91 \pm 1.66$ & 0.171 \\
(number) & & & \\
\hline
\end{tabular}

A significant deviation towards male sex preference was found in patients of Chinese, Indian and Middle Eastern ethnic background. In our study, the embryonic sex ratio was found to be $1 .{ }^{17}$ This reported that the sex ratio at both fertilization and implantation is between 1.29, 1.50 and 1.07 for PGD, IVF and ICSI cycles, respectively. ${ }^{18}$

In another study, the effect of male age on the spermsex ratio was studied. ${ }^{19}$ They observed a significant difference between live birth and sperm-sex ratio $(p<0.0001)$. However, the finding of our study did not support this finding.

Panahi and Fahami in the year 2015 studied the result of pre-implantation genetic diagnosis in relation to couples' age. ${ }^{20}$ Their result suggested no significant relationship between the age of the patient and the rate of chemical and clinical pregnancy and gestational weight of the newborn. However, the PGD method was $100 \%$ successful in achieving the desired sex.
It is worth mentioning that, in non-medical PGD sex selections, we perform IVF treatment cycles in spite of the fact that most of these cases have no fertility problems, since it is the only method where we can select the desired embryos. At the same time, we face a lot of problems in treating infertility cases (endometriosis, poor responders, low AMH) and this will reduce the chances of selecting embryos. ${ }^{21}$ These cases will be dealt with by collecting embryos sometimes from more than one IVF cycle. Although these cases are limited in number, they represent a great challenge. On the other hand, cases with PCOS infertility may show a risk of hyperstimulation syndrome and might increase the chances of cancellation or freezing all the embryos. Also, in these patients it is difficult to optimize the dose of recombinant follicle-stimulating hormone (FSH) needed for stimulation. ${ }^{22}$

Knowing the gender proportion in couples who have offspring of a particular gender can help the physician counseling the couples identify the probability of achieving the desired embryo. This study revealed the fact that the couples who predominately produce the same gender (females in our study) are able to produce embryos of the opposite gender as well. However, the ratio of the same embryos is higher than that of the opposite embryo. This is a clear statement that can be provided during counseling sessions before the intended PGD sex selection.

\section{Conclusion}

A greater variability in the female to male embryonic ratio is produced in couples having predominantly female offspring and seeking non-medical PGD for male sex selection.

Table 3 Chi-Square Test (for Total Embryos)

\begin{tabular}{|c|c|c|c|c|c|}
\hline & & & \multicolumn{2}{|c|}{ Embryo Ratio } & \multirow[t]{2}{*}{ p-value } \\
\hline & & & Unequal Ratio & Equal Ratio & \\
\hline \multirow[t]{2}{*}{ Pregnancy outcome } & Negative & $\begin{array}{l}\mathrm{N} \\
\% \text { within pregnancy outcome }\end{array}$ & $\begin{array}{l}43 \\
86.0 \%\end{array}$ & $\begin{array}{l}7 \\
14.0 \%\end{array}$ & \multirow[t]{2}{*}{0.843528} \\
\hline & Positive & $\begin{array}{l}\mathrm{N} \\
\% \text { within pregnancy outcome }\end{array}$ & $\begin{array}{l}44 \\
84.6 \%\end{array}$ & $\begin{array}{l}8 \\
15.4 \%\end{array}$ & \\
\hline \multirow[t]{2}{*}{ Type of protocol } & Short & $\begin{array}{l}\mathrm{N} \\
\% \text { within type of protocol }\end{array}$ & $\begin{array}{l}96 \\
85.0 \%\end{array}$ & $\begin{array}{l}17 \\
15.0 \%\end{array}$ & \multirow[t]{2}{*}{1.0} \\
\hline & Long & $\begin{array}{l}\mathrm{N} \\
\% \text { within type of protocol }\end{array}$ & $\begin{array}{l}2 \\
100.0 \%\end{array}$ & $\begin{array}{l}0 \\
0.0 \%\end{array}$ & \\
\hline
\end{tabular}




\section{Data Sharing Statement}

The authors confirm that the data supporting the findings of this study are available within the article.

\section{Acknowledgments}

Many thanks to the Research Faculty at Jordan University of Science and Technology and Sulfa Khaled Selo.

\section{Funding}

The authors declare that they have not received any funds from any source.

\section{Disclosure}

No potential conflicts of interest were reported by the authors.

\section{References}

1. Handyside A, Konotogianni EH, Hardy K, et al. Pregnancies from biopsied human pre-implantation embryos sexed by Y-specific DNA amplify-cation. Nature. 1990;344:768-770. doi:10.1038/344768a0

2. Kirshenbaum M, Feldman B, Aizer A, et al. Preimplantation embryos sex ratios in couples with four or more children of same sex, what should be expected from a preimplantation genetic diagnosis cycle? Gynecol Endocrinol. 2019;35(6):515-517. doi:10.1080/ 09513590.2018.1563886

3. Werlin L, Rodi I, DeCherney A, et al. Preimplantation genetic diagnosis (PGD) as both a therapeutic and diagnostic tool in assisted reproductive technology. Fertil Steril. 2003;80(2):467-468. doi:10.1016/S0015-0282(03)00605-8

4. The Preimplantation Genetic Diagnosis International Society PGDIS. Guidelines for good practice in PGD: programme requirements and laboratory quality assurance. Reprod Biomed. 2008;16(1):134-147. doi:10.1016/S1472-6483(10)60567-6

5. Wilton L, Thornhill A, Traeger-Synodinos J, et al. The causes of misdiagnosis and adverse outcomes in PGD. Hum Reprod. 2009;24 (5):1221-1228. doi:10.1093/humrep/den488

6. Lewis CM, Pinel T, Whittaker JC, et al. Controlling misdiagnosis errors in pre-implantation genetic diagnosis: a comprehensive model encompassing extrinsic and intrinsic sources of error. Hum Reprod. 2001;16(1):43-50. doi:10.1093/humrep/16.1.43

7. Piyamongkol W, Bermudez MG, Harper JC, et al. Detailed investigation of factors influencing amplification efficiency and allele drop-out in single cell PCR: implications for preimplantation genetic diagnosis. Mol Hum Reprod. 2003;9(7):411-420. doi:10.1093/molehr/gag051
8. DeWert G, Dondorp W. Preconception sex selection for non-medical and intermediate reasons: ethical reflections. Facts Views Vis Obgyn. 2010;2(4):267-277.

9. Strange H. Non-medical sex selection: ethical issues. $\mathrm{Br}$ Med Bull. 2010;94:7-20. doi:10.1093/bmb/ldq002

10. Klitzman R, Abbate KJ, Chung WK, et al. Views of pre-implantation genetic diagnosis among psychiatrists and neurologists. $J$ Reprod Med. 2015;59(7-8):385-392.

11. Westoff CF, Rindfuss RR. Sex preselection in the United States: some implications. Science. 1974;184(4137):633-636. doi:10.1126/ science.184.4137.633

12. Steinbacher R, Gilroy FD. Preference for sex of child among primiparous women. $J$ Psychol. 1985;119(6):541-547. doi:10.1080/ 00223980.1985.9915461

13. ACOG Committee. ACOG committee opinion no. 360: sex selection. Obstet Gynecol. 2007;109(2 Pt 1):475-478. doi:10.1097/00006250200702000-00063

14. Mukherjee T, Flisser E, Copperman AB, et al. Preimplantation genetic diagnosis for gender selection: you don't always get what you want. Open J Obstet Gynecol. 2012;2:291-297. doi:10.4236/ ojog.2012.23062

15. Colls P, Silver L, Olivera G, et al. Preimplantation genetic diagnosis for gender selection in the USA. Reprod Biomed Online. 2009;19 (2):16-22. doi:10.1016/S1472-6483(10)60273-8

16. Alamri A, Nam JY, Blancato JK. Fluorescence in situ hybridization of cells, chromosomes, and formalin-fixed paraffin-embedded tissues. Methods Mol Biol. 2017;1606:265-279.

17. Feldman B, Aizer A, Brengauz M, et al. Preimplantation genetic diagnosis should we use ICSI for all? J Assist Reprod Genet. 2017;34(9):1179-1183. doi:10.1007/s10815-017-0966-7

18. Tarın J, Garcia-Perez MA, Hermenegildo C, et al. Changes in sex ratio from fertilization to birth in assisted-reproductive-treatment cycles. Reprod Biol Endocrinol. 2015;12:56. doi:10.1186/14777827-12-56

19. Graffelman J, Fugger EF, Keyvanfar K, et al. Human live birth and sperm-sex ratios compared. Hum Reprod. 1999;14(11):2917-2920. doi:10.1093/humrep/14.11.2917

20. Panahi S, Fahami F. The results of pregnancies after gender selection by pre implantation genetic diagnosis and its relation with couple's age. Iran J Nurs Midwifery Res. 2015;20(6):670-675. doi:10.4103/ 1735-9066.170012

21. Šalamun V, Verdenik I, Laganà AS, Vrtačnik-Bokal E. Should we consider integrated approach for endometriosis-associated infertility as gold standard management? Rationale and results from a large cohort analysis. Arch Gynecol Obstet. 2018;297(3):613-621. doi:10.1007/s00404-017-4633-0

22. Papler TB, Bokal EV, Zmrzljak UP, et al. PGR and PTX3 gene expression in cumulus cells from obese and normal weighting women after administration of long-acting recombinant follicle-stimulating hormone for controlled ovarian stimulation. Arch Gynecol Obstet. 2019;299(3):863-871. doi:10.1007/s00404018-5031-y
Risk Management and Healthcare Policy is an international, peerreviewed, open access journal focusing on all aspects of public health, policy, and preventative measures to promote good health and improve morbidity and mortality in the population. The journa welcomes submitted papers covering original research, basic science, clinical \& epidemiological studies, reviews and evaluations, guidelines, expert opinion and commentary, case reports and extended reports. The manuscript management system is completely online and includes a very quick and fair peer-review system, which is all easy to use. Visit http://www.dovepress.com/testimonials.php to read real quotes from published authors. 